COMENTARIOS DE JURISPRUDENCIA

\title{
La jurisprudencia andina y europea en materia de protección del software, las aplicaciones móviles y los videojuegos
}

\author{
Andean and European case law on the protection of software, \\ mobile applications and video games
}

\author{
Juan Manuel Indacochea Jauregui \\ Pontificia Universidad Católica del Perú
}

\begin{abstract}
RESUMEN La jurisprudencia andina y europea en materia de protección del software ha tenido un desarrollo paralelo a ambas normativas comunitarias. Si bien las decisiones o directivas comunitarias establecen la protección del software por derecho de autor, es posible la patentabilidad de las denominadas invenciones patentadas por ordenador, bajo ciertas condiciones que serán analizadas en el presente estudio. Adicionalmente, se explicará el tratamiento jurisprudencial de las obras audiovisuales, como los videojuegos, tanto en la Comunidad Andina como en la Unión Europea.
\end{abstract}

PALABRAS CLAVE Aplicaciones, Comunidad Andina, programa de ordenador, Tribunal Andino, Unión Europea, videojuegos.

ABSTRACT The Andean and European case law on software protection has developed parallel to both community regulations. Although community decisions or directives establish the protection of software by copyright, software patents are possible, under certain conditions that will be analyzed in this study. Additionally, the jurisprudential treatment, in the Andean Community and the European Union, of audiovisual works, such as video games, will be explained.

KEYWORDS Applications, Andean Community, software, Andean Court of Justice, European Union, videogames. 


\section{Introducción}

En virtud del distanciamiento social se suele pasar más tiempo conectado a Internet que antes. Durante los últimos años, entre las industrias con mayor crecimiento se encuentran aquellas pertenecientes al rubro de la tecnología de la información (tecnología de la computación). La tecnología de la información abarca áreas de diversa índole como hardware, software, aplicaciones, bases de datos, seguridad informática (ciberseguridad), sistemas y redes informáticas en general (por ejemplo, redes LAN, MAN o WAN, como el Internet). ${ }^{1}$ Esta creciente industria tiene actualmente una capitalización bursátil de 20.000 millones de dólares y representa la cuarta parte del valor total del mercado bursátil a nivel mundial. ${ }^{2}$

Se espera que en 2020 las aplicaciones de telefonía móvil generen por lo menos 189 mil millones de dólares de ingresos. ${ }^{3}$ Las empresas de software —en general- tuvieron ingresos por 466.800 millones de dólares solo en 2019. Inicialmente, en 2020 , el mercado global de software preveía ingresos por 487.180 millones de dólares, ${ }^{4} \mathrm{ex}$ pectativa que probablemente será superada si se tiene en consideración la vigencia de la pandemia de la Covid-19, enfermedad causada por el virus SARS-CoV-2.

A manera de referencia, los ingresos de la industria del software inicialmente previstos para el 2020 superarían ampliamente el PIB de Argentina, el cual fue apenas superior a los 400 mil millones de dólares en 2019. ${ }^{5}$ El PIB de Perú, por ejemplo, fue de alrededor de 200 mil millones de dólares en $2018 ;{ }^{6}$ mientras que el PIB de Chile se mantuvo por encima de los 250 mil millones de dólares durante 2018 y 2019.7

El presente estudio tiene como objetivo reseñar el marco jurídico de protección del software en la Comunidad Andina (CAN). Ante todo, es preciso aclarar ciertos conceptos fundamentales a fin de vislumbrar una solución interpretativa a la pro-

1. «What is information technology or IT? Definition and examples», Market Business News, disponible en https://bit.ly/35TTVyC.

2. «TikTok and the Sino-American tech split», The Economist,9 de julio de 2020, disponible en https:// econ.st/32NUKXN.

3. Ian Blair, «Mobile App Download and Usage Statistics (2020», Build Fire, disponible en http://bit. ly/3aJ 5 Odc.

4. «Software market revenue in the World from 2016 to 2021 (in billion U.S. Dollars)», Statista, disponible en https://bit.ly/2ZUgX4A.

5. El PIB de Argentina fue de 402.273 millones de dólares. «PIB de Argentina», Expansión, disponible en http://bit.ly/3mOnVRi y Banco Mundial, «The World Bank in Argentina», disponible en http://bit. ly/zaGKepW.

6. Banco Mundial, «The World Bank in Peru», disponible en http://bit.ly/34CAZDh y «PIB de Perú», Expansión, disponible en http://bit.ly/2M9IwDj.

7. El PIB de Chile fue de 252.035 millones de dólares en 2019 y de 252.481 millones de dólares en 2018. «PIB de Chile», Expansión, disponible en http://bit.ly/3nMbHdu y Banco Mundial, «The World Bank in Chile», disponible en http://bit.ly/2WGgUY7. 
blemática en relación con la falta de referencia expresa a las aplicaciones móviles, a propósito de la protección conferida a los creadores o diseñadores de software y videojuegos a nivel nacional y subregional.

\section{Conceptos fundamentales}

Software es un término que hace referencia a un programa o conjunto de programas informáticos, así como datos, procedimientos y pautas que permiten realizar distintas tareas en un sistema informático. La Real Academia Española define software como el «conjunto de programas, instrucciones y reglas informáticas para ejecutar ciertas tareas en una computadora». Según el estándar 729 del Instituto de Ingeniería Eléctrica y Electrónica (IEEE, por sus siglas en inglés), es el conjunto de los programas de cómputo, procedimientos, reglas, documentación y datos asociados, que forman parte de las operaciones de un sistema de computación (IEEE, 1993).

Los dos tipos de software que generalmente es posible encontrar en toda computadora o dispositivo móvil son de sistema y de aplicación. Software de sistema es aquel programa responsable de la ejecución de las aplicaciones necesarias para que un sistema opere correctamsente. Los sistemas operativos más populares para computadoras son Microsoft Windows, macOS y Linux; mientras que los principales sistemas operativos móviles son iOS (Apple) y Android (Google).

El software de aplicación —o simplemente aplicación- es un programa diseñado a fin de facilitar tareas específicas para un determinado medio informático. Una aplicación móvil o app (derivado del inglés application) es un software de aplicación que se puede descargar y al que se puede acceder directamente desde un dispositivo móvil. ${ }^{8}$

En consecuencia, el concepto de programa de ordenador, también denominado programa informático (de computación), comprende tanto los sistemas operativos como las aplicaciones en general, incluyendo aquellas diseñadas para dispositivos móviles (sistemas operativos móviles y aplicaciones móviles o apps).

Es preciso advertir que, si bien los dispositivos móviles más conocidos son los teléfonos inteligentes (smartphones) y las tabletas (tablets), existen también televisores inteligentes (Smart TVs), vehículos, reproductores $\mathrm{MP}_{3}$ y $\mathrm{MP}_{4}$, así como wearable technology, como por ejemplo rastreadores de ejercicios (fitness trackers), relojes inteligentes (smartwatches), cascos de realidad virtual (VR headsets), lentes de realidad aumentada o lentes inteligentes (smartglasses) y toda clase de dispositivos para la salud del consumidor. ${ }^{9}$

8. Comisión Federal de Comercio, «Aplicaciones móviles: Qué son y cómo funcionan», disponible en http://bit.ly/38Noc2f.

9. «What is wearable tech? Everything you need to know explained», Wareable, 17 de agosto de 2019, disponible en https://bit.ly/35Uxk53. 


\section{La protección conferida en la Comunidad Andina}

En el ámbito de la CAN, rige lo establecido en el artículo 4 literal l) de la Decisión 351, ${ }^{10}$ adoptada en 1993 por la Comisión del Acuerdo de Cartagena (ahora Comisión de la Comunidad Andina); la cual guarda armonía con lo dispuesto en el artículo 4 del Tratado de la Organización Mundial de la Propiedad Intelectual (OMPI) sobre Derecho de Autor y con el artículo 10 del Acuerdo sobre los Aspectos de los Derechos de Propiedad Intelectual relacionados con el Comercio (Acuerdo sobre los ADPIC) de la Organización Mundial del Comercio (OMC).

Es preciso destacar que en la definición contenida en el vigésimo párrafo del artículo 3 de la Decisión 351 se equipara el concepto de programa de ordenador con el de software. Adicionalmente, el órgano jurisdiccional supranacional de la CAN, el Tribunal de Justicia de la Comunidad Andina (TJCA), incluso con anterioridad a la adopción de la Decisión 486, también equiparaba el concepto de software con el de programa de ordenador. ${ }^{11}$ Además, en recientes interpretaciones prejudiciales, el TJCA afirmó que el programa de ordenador es «llamado comúnmente software» (TJCA, 2019: 4). Por consiguiente, en el sistema subregional andino los términos software y programa de ordenador son intercambiables, opción normativa que puede entenderse desde una perspectiva práctica toda vez que comúnmente se emplea el anglicismo software para referirse a programas informáticos en general.

Mientras que el artículo 15 literal e) de la Decisión $486^{12}$ excluye expresamente de la materia patentable «los programas de ordenadores o el soporte lógico, como tales»; el artículo 4 literal l) de la Decisión 351 los incluye en el listado abierto de obras susceptibles de protección por el derecho de autor. Asimismo, el primer párrafo del artículo 23 de la Decisión 351 dispone que estos «se protegen en los mismos términos que las obras literarias», precisando a continuación que «dicha protección se extiende tanto a los programas operativos como a los programas aplicativos».

En virtud de lo previsto en el artículo 23 de la Decisión 351, el TJCA clasifica igualmente los programas en operativos (de sistema) y aplicativos (de aplicación) (TJCA , 2018b: 4, para. 1.6). Cabe mencionar que, de manera similar, en la Unión Europea, el Considerando (7) de la Directiva 2009/24/CE del Parlamento Europeo y del Consejo de 23 de abril de 2009 sobre la protección jurídica de programas de ordenador prevé que: «el término "programa de ordenador" incluye programas en cualquier forma».

Los programas informáticos o de ordenador son protegidos en los países miem-

10. Comisión del Acuerdo de Cartagena, Decisión 351, Régimen Común sobre Derecho de Autor y Derechos Conexos, 17 de diciembre de 1993.

11. «En efecto, el "software" o programa de ordenador, es definido por el artículo 3 de la Decisión 351 como». Compárese TJCA (1999: 5 y 2005: 9).

12. Comisión de la Comunidad Andina, Decisión 486, Régimen Común sobre Propiedad Industrial, 14 de septiembre de 2000 . 
bros de la CAN por el derecho de autor desde su creación. Los derechos de un autor sobre su obra nacen ipso facto en el momento mismo de su creación. Si bien el registro no es necesario a fin de obtener dicha protección legal, es recomendable a efectos de contar con una fecha cierta de creación del programa.

Es importante advertir que la protección del software por el derecho de autor conlleva a que ésta se encuentre supeditada al cumplimiento del requisito esencial - para la protección de obras literarias o artísticas- de la originalidad. El objeto de protección jurídica es la expresión particular de una idea (forma), no la idea misma. Por lo tanto, la principal desventaja de la protección conferida por el derecho de autor radica en que no se protege la funcionalidad o técnica del programa informático. En otras palabras, otro programador puede desarrollar un software que cumpla la misma función o puede aplicar la técnica de un programa protegido a otro problema técnico de una manera diferente sin infringir los derechos de autor de su titular.

En contrapartida, una ventaja podría ser la mayor duración de la protección por el derecho de autor. En efecto, de conformidad con el artículo 18 de la Decisión 351, la duración de la protección no será inferior a la vida del autor y cincuenta años post mortem. ${ }^{13}$ Sin embargo, cabría preguntarse qué tan relevante resulta esta aparente ventaja en la actual era digital o informática.

Al definir el concepto de programa de ordenador, el TJCA indica reiteradamente que la protección conferida abarca los siguientes elementos del software: i) el código fuente; ii) el código objeto (o ejecutable); iii) la documentación técnica (por ejemplo, memoria descriptiva); y, iv) los manuales de uso o usuario. ${ }^{14}$ De esta manera, el órgano jurisdiccional de la CAN explica el concepto de programa de ordenador o software, mencionando asimismo sus componentes (TJCA, 2018a: 5, para. 1.4):

Se entiende por programa de ordenador o software a la expresión de un conjunto de instrucciones mediante palabras, códigos, planes o en cualquier otra forma que, al ser incorporadas en un dispositivo de lectura automatizada, es capaz de hacer que un ordenador - un aparato electrónico o similar capaz de elaborar informaciones-, ejecute determinada tarea u obtenga determinado resultado. Asimismo, el programa de ordenador comprende también la documentación técnica y los manuales de uso; ${ }^{15}$

13. Por ejemplo, en Perú, en concordancia con la disposición comunitaria, el artículo 52 del Decreto Legislativo 822 establece que el derecho de autor dura toda la vida del autor y setenta años después de su fallecimiento; mientras que el artículo 54 dispone a su vez que «en las obras colectivas, los programas de ordenador, las obras audiovisuales, el derecho patrimonial se extingue a los setenta años de su primera publicación o, en su defecto, al de su terminación».

14. Adicionalmente, se protegen por derecho de autor las versiones sucesivas del programa de ordenador, los diferentes módulos que puedan componer el programa informático y los programas que puedan derivarse del mismo.

15. De conformidad con la definición establecida en el artículo 3 de la Decisión 351 de la Comisión del Acuerdo de Cartagena. 
es decir, los elementos del programa de ordenador que quedan protegidos son: el código fuente, el código objeto (o ejecutable), la documentación técnica (por ejemplo, memoria descriptiva) y los manuales de uso o usuario.

En relación con la patentabilidad del programa de ordenador o software, es preciso comentar las denominadas invenciones implementadas por ordenador o "patentes de software», es decir, aquellas invenciones que requieren la utilización de un ordenador, red informática u otro aparato programable en los que la ejecución de por lo menos un programa informático produce un efecto técnico que forma parte de la solución al problema técnico planteado. ${ }^{16}$ En general, no son patentables aquellas invenciones implementadas por ordenador que no producen ningún efecto técnico, además de la normal interacción física entre un determinado programa y el correspondiente ordenador, red o aparato programable en que se ejecute (Indecopi, 2019: 8).

En términos generales, se considera que una invención implementada por ordenador tiene un efecto técnico si: i) afecta el funcionamiento físico o técnico de un dispositivo; ii) procesa datos que son parámetros operativos de un dispositivo y de cuyo resultado depende el funcionamiento técnico de dicho dispositivo; iii) produce un efecto técnico como resultado de la interrelación entre el ordenador y el mundo exterior; o, iv) en general, si resuelve un problema técnico (Indecopi, 2019: 9).

Es importante señalar que, si bien la Decisión 351 podría no prever todos los posibles conflictos y aplicaciones relacionados con el derecho de autor toda vez que fue adoptada en 1993, el TJCA ha colmado ciertos vacíos del ordenamiento comunitario andino. Así, por ejemplo, el órgano jurisdiccional comunitario ha desarrollado las particularidades jurídicas de las obras multimedia. De esta manera, se refirió a los videojuegos con ocasión del fallo pronunciado en el marco del Proceso 464-IP-2017 (caso Nintendo) (TJCA, 2018a: 65-66, paras. 1.34-1.36):

1.34. Por ejemplo, un videojuego (obra multimedia) al igual que una obra audiovisual (sea una novela, serie de televisión, películas, spots publicitarios, videoclips, etc.) está conformada por imágenes asociadas que dan la sensación de movimiento, así como también por un argumento, obras artísticas (dibujos de personajes, paisajes), obras musicales, etc., las cuales podrán ser visualizadas a través de un aparato idóneo como pudiera ser una pantalla de televisor o computador, tabletas, laptop, visor de realidad virtual, consola de juegos o cualquier otro aparato conocido o por conocerse. No obstante a lo anterior, la diferencia radicará en que una obra multimedia siempre permitirá la interacción entre el usuario y la creación multimedia (en el

16. Oficina Europea de Patentes, «Guidelines for Search and Examination at the EPO as PCT Authority», parte-G-II, 3.6., disponible en http://bit.ly/38vlKx7. Véase también Superintendencia de Industria y Comercio, «¿Por qué se patentan las invenciones implementadas por computador?», disponible en https://bit.ly/38oGgqA. 
ejemplo, el videojuego), lo cual no sucede en una obra audiovisual. Asimismo, para que exista la interacción antes señaladas, necesariamente se requerirá la ejecución de un programa de ordenador, situación que tampoco es prescindible en las obras audiovisuales. [...]

1.36. Asimismo, resulta necesario indicar que los elementos originales que pudieran formar parte de la obra multimedia - dibujos de personajes (obra artística), música (obra musical), argumentos y guiones (obras literarias), etc. - se encuentran igualmente protegidos por la legislación del derecho de autor e incluso sus autores pueden explotarlos económicamente de manera independiente a la obra multimedia, salvo que se haya estipulado lo contrario.

En consecuencia, el TJCA - al igual que el Tribunal de Justicia de la Unión Europea (TJUE, 2014) - considera que los videojuegos constituyen una especie de obra multimedia y como tal se encuentra conformada por diferentes elementos (por ejemplo, musicales, argumentos, guiones, dibujos, etcétera), los cuales a su vez pueden encontrarse protegidos por derechos de autor o por derechos conexos (por ejemplo, interpretaciones musicales o fonograma) que podrían ser titularidad de terceros.

\section{La jurisprudencia europea}

En la Unión Europea, continúa rigiendo la Directiva 2009/24/CE del Parlamento Europeo y del Consejo de 23 de abril de 2009 sobre la protección jurídica de programas de ordenador, cuyo artículo 1 prevé, al igual que la norma andina, la protección de los programas de ordenador (software) por medio del derecho de autor, en los mismos términos que las obras literarias. Asimismo, se advierte que las ideas y principios subyacentes de un programa, incluyendo aquellos relacionados con su interfaz, no se encuentran protegidos por el derecho de autor, lo cual responde a la propia naturaleza del derecho de autor, mediante el cual no se protegen las ideas propiamente dichas, sino la forma en que estas se plasman.

Además, el numeral 2 del artículo 2 del Convenio de Múnich sobre Concesión de Patentes Europeas ${ }^{17}$ enumera una lista no limitativa de exclusiones a la patentabilidad que comprende los programas de ordenador. Sin embargo, el numeral 3 del citado artículo establece como condición de la exclusión que la solicitud o «la patente europea no se refiera más que a uno de esos elementos considerado como tal».

Adicionalmente, del numeral 1 del citado artículo 52 del Convenio de Múnich se deriva el requisito del «carácter técnico» de la invención. En ese sentido, las Directrices de Examen de la Oficina Europea de Patentes (OEP) admiten la patentabilidad de procedimientos que utilicen un programa en la medida que se obtenga un «efecto

17. Convenio de Múnich sobre Concesión de Patentes Europeas, de 5 de octubre de 1973; modificado por el Acta de revisión de 29 de noviembre de 2000, que entró en vigor el 13 de diciembre de 2007. 
técnico». ${ }^{18}$ Un ejemplo del referido criterio de la OEP lo constituye la decisión de la Sala de Apelación de 21 de abril de 2004, recaída en el asunto T 258/03, comúnmente conocido en Francia como el célebre caso Hitachi, en la cual estableció que un método llevado a cabo por computadora podría tener un «carácter técnico» si logra obtener un «efecto técnico»; lo cual no se habría cumplido en ese caso concreto toda vez que se trataría de una «simple manifestación de informaciones» bien conocida en el ámbito del manejo de datos. ${ }^{19}$

Desde la década de los 8o, en virtud de lo previsto por el Convenio de Múnich y las Directrices de Examen de la OEP, las salas de apelación de la OEP han admitido la patentabilidad de software siempre que se cumpliera —además de los requisitos de novedad, nivel inventivo y aplicación industrial - con la condición esencial del resultado o «efecto técnico» de modo de ser considerado como una invención en decisiones importantes, tales como Vicom, Xray Apparatus, IBM, Sohei, Petterson y Queueing. ${ }^{20}$

En particular, cabe destacar la decisión recaída en el asunto T 1173/97, uno de los casos que involucró a IBM, en la cual la Sala de apelación advirtió que el artículo 10 del Acuerdo sobre los ADPIC de la OMC, que prevé la protección del software (programas de ordenador) por el derecho de autor, no excluye la protección por el derecho de patentes, teniendo en cuenta la redacción del artículo 27(1), relativo a la materia patentable, el cual dispone que se pueden conceder invenciones «en todos los campos de la tecnología» siempre que se cumplan los requisitos de patentabilidad.

Por otra parte, cabe destacar que el Considerando (7) de la Directiva 2009/24/ $\mathrm{CE}$ indica que: «A los efectos de la presente Directiva, el término "programa de ordenador" incluye programas en cualquier forma». Por consiguiente, la protección otorgada en la Unión Europea alcanza a las aplicaciones móviles o apps, así como al software «incorporado en el hardware» de dispositivos como consolas de videojuegos, televisores, relojes inteligentes, entre otros.

En 2019, se admitió a trámite un caso que enfrenta a Google y a la Comisión Euro-

18. Oficina Europea de Patentes, «Guidelines for Examination in the European Patents Office», parteC, disponible en http://bit.ly/38Cbx1K.

19. Oficina Europea de Patentes, Sala de Apelación Técnica (Chambre de recours technique), decisión de 21 de abril de 2004, asunto T 258/03 (demandante: Hitachi, Ltd.), «Motivos de la decisión», apartado 5.4, tercer párrafo, p. 8. El referido caso «Hitachi» ha sido citado en más de una centena -más de 120- de decisiones de las salas de apelación de la OEP. Véase Oficina Europea de Patentes, Sala de Apelación, T 0258/03 (Auction method/HITACHI), 21 de abril de 2004, disponible en http://bit.ly/3nRodbq.

20. Véase, por ejemplo, las decisiones pronunciadas en el marco del asunto T 208/84, caso Vicom, sobre una invención relacionada con computadora, 1987, p. 14; asunto T 26/86, sobre un equipo radiológico (Xray Apparatus), de 21 de mayo de 1987; asunto T 110/90, caso IBM, sobre una forma de documento editable, 1994; asunto T 769/92, caso Sohei, sobre un sistema de gestión universal, de 31 de mayo de 1994; asunto T 1002/92, caso Petterson, sobre el sistema Queueing, de 6 de julio de 1994; entre otros. 
pea por un presunto caso de abuso de posición dominante. ${ }^{21}$ La posición dominante - en este caso en particular- ${ }^{22}$ estaría determinada por la titularidad del derecho de autor sobre el sistema operativo Android, mientras que el supuesto abuso consistiría en exigir licencias - a los productores de smartphones o vendedores finales- no solo por el uso - instalación- del sistema operativo sino por las apps de los accesorios y servicios complementarios.

La reciente aprobación de la Directiva (UE) 2019/790 $0^{23}$ sobre los derechos de autor en el mercado único digital en 2019 constituye un gran avance en comparación con la normativa andina vigente (la Decisión 351 es del año 1993). Cabe mencionar que el artículo 23 numeral 2 de la referida Directiva establece que: «Los Estados miembros dispondrán que los artículos 18 a 22 de la presente Directiva no se apliquen a los autores de un programa de ordenador en el sentido del artículo 2 de la Directiva 2009/24/CE».

El Tribunal de Justicia de la Unión Europea (TJUE) puso en evidencia las limitaciones de la protección del software o programas de ordenador por el derecho de autor en el primer párrafo del pronunciamiento de la Sentencia recaída en el asunto C-406/10:

El artículo 1, apartado 2, de la Directiva 91/250/CEE del Consejo, de 14 de mayo de 1991, sobre la protección jurídica de programas de ordenador, debe interpretarse en el sentido de que ni la funcionalidad de un programa de ordenador ni el lenguaje de programación o el formato de los archivos de datos utilizados en un programa de ordenador para explotar algunas de sus funciones constituyen una forma de expresión de ese programa y, por ello, carecen de la protección del derecho de autor sobre los programas de ordenador en el sentido de esa Directiva. ${ }^{24}$

En relación con la protección conferida a los videojuegos, el TJUE declaró, en el párrafo 23 de la sentencia recaída en el asunto C-355/12 (caso Nintendo c/ PC Box), lo siguiente (TJUE, 2014):

23 No desvirtúa esta constatación el hecho de que la Directiva 2009/24 sea lex specialis en relación con la Directiva 2001/29 (véase la sentencia de 3 de julio de

21. Tribunal de Justicia de la Unión Europea, Tercera Sala del Tribunal General, asunto T-604/18, Google LLC y Alphabet Inc. c/ Comisión Europea, Orden de 23 de septiembre de 2019, disponible en http:// bit.ly/38sNHFK.

22. Dellana, Alessio, «Google appeal against $€ 2.4 \mathrm{bn}$ fine takes place at European Court of Justice», EuroNews, 14 de febrero de 2020. Disponible en http://bit.ly/3nOBm5f.

23. Parlamento Europeo y Consejo Europeo, Directiva (UE) 2019/79o sobre los derechos de autor y derechos afines en el mercado único digital y por la que se modifican las Directivas 96/9/CE y 2001/29/ CE, 17 de abril de 2019 .

24. Tribunal de Justicia de la Unión Europea, sentencia del asunto C-406/10, caso SAS Institute Inc. c/ World Programming Ltd, 2 de mayo de 2012. 
2012, UsedSoft, C-128/11, apartado 56). En efecto, de conformidad con su artículo 1, apartado 1, la protección que ofrece la Directiva 2009/24 se limita a los programas de ordenador. Ahora bien, como indica la resolución de remisión, los videojuegos, tales como los controvertidos en el litigio principal, son un material complejo que incluye no sólo un programa de ordenador, sino también elementos gráficos y sonoros que, aunque codificados en lenguaje informático, tienen un valor creativo propio que no puede reducirse a dicha codificación. En la medida en que las partes de un videojuego, en el presente caso esos elementos gráficos y sonoros, participan de la originalidad de la obra, están protegidas, junto con el conjunto de la obra, por los derechos de autor en el régimen establecido por la Directiva 2001/29. ${ }^{25}$

En consecuencia, en la Unión Europea, al igual que en la CAN, se considera que los videojuegos no se reducen al concepto de programa de ordenador, sino que pueden comprender además elementos (gráficos, sonoros o de otra índole) susceptibles de ser igualmente protegidos por derechos de autor y derechos conexos de manera individual. Al respecto, cabe mencionar que, generalmente, dichas obras audiovisuales, musicales, artísticas o literarias reproducidas en los videojuegos suelen tener autores o titulares diferentes.

En Francia, por mencionar un país miembro de la Unión Europea, la jurisprudencia ha reconocido desde los años 80 que un software puede ser patentable ante la eventualidad de un resultado final técnico de su utilización..$^{26}$ Cabe precisar que, tanto los tribunales franceses como la autoridad administrativa competente (Institut national de la propriété industrielle - INPI), consideran que la simple utilización de medios técnicos conocidos - como el Internet- no pueden conferir a la invención reivindicada el carácter técnico requerido ${ }^{27}$ de lo cual se puede inferir que se trata de un requisito relativo al resultado obtenido («efecto técnico»), no a los medios empleados.

\section{Conclusiones}

El programa de ordenador o software es protegido por medio del derecho de autor en los demás países miembros de la CAN. En el sistema subregional andino, los términos software y programa de ordenador son intercambiables. La normativa comunitaria andina clasifica el software o los programas de ordenador en operativos (de sistema) y aplicativos (de aplicación), comprendiendo a ambos dentro de su ámbito

25. Tribunal de Justicia de la Unión Europea, sentencia del asunto C-355/12, caso Nintendo c/ PC Box, 23 de enero de 2014.

26. Véase, por ejemplo, las Sentencias de la Corte de Casación de París recaídas en el caso Schlumberger, de 15 de junio de 1981, y en el caso Gachet c. Taxicop, de 18 de febrero de 2004.

27. Véase, por ejemplo, Corte de Apelación de Rennes (Cour d'Appel de Rennes), Sentencia de 7 de octubre de 2003, caso Antonietti c. INPI, que confirma la decisión del INPI. 
de aplicación. Las aplicaciones móviles o apps se encuentran igualmente dentro del ámbito de aplicación de la norma andina toda vez que están comprendidas de manera implícita dentro del concepto de programas aplicativos (de aplicación).

Toda vez que el objeto de protección del derecho de autor es la expresión particular de una idea, no la idea misma; la protección por derecho de autor no abarca la funcionalidad o técnica del programa informático, protegiendo únicamente la forma en que se encuentra expresada la obra (código fuente, código ejecutable; documentación técnica y manuales de usuario).

En el sistema subregional andino, tanto para la normativa comunitaria andina como para el TJCA, los términos «software» $\mathrm{y}$ «programa de ordenador» son intercambiables. Además, dicho concepto comprende el software de sistema y el de aplicación, incluyendo las aplicaciones móviles o apps. Una situación similar ocurre en la Unión Europea, donde la aún vigente Directiva 2009/24/CE se encuentra expresamente consagrada a «la protección jurídica de programas de ordenador». La opción legislativa de emplear las palabras «programa de ordenador» constituye la alternativa, en idioma español, al anglicismo «software», utilizado para referirse a programas informáticos en general.

Con la reciente aprobación de la Directiva (UE) 2019/790 sobre los derechos de autor y derechos afines en el mercado único digital, la normativa comunitaria europea llevaría algo de ventaja a la andina. ${ }^{28}$ En efecto, la Decisión 351 data del año 1993 , por lo que comprensiblemente no habría anticipado el advenimiento de la era digital. Por consiguiente, cabría plantear la necesidad de revisar —en virtud del carácter dinámico del derecho - una normativa que probablemente haya quedado desfasada al no anticipar situaciones que han venido surgiendo en el entorno digital.

A este respecto, el propio Parlamento Europeo reconoce que: «La legislación relativa a los derechos de autor y los derechos afines a la evolución tecnológica [...] ahora está desfasada con respecto a la evolución vertiginosa registrada en el mundo digital». ${ }^{29}$ Lo mismo sería aplicable, con mayor razón, a la normativa comunitaria andina y, consecuentemente, a las legislaciones nacionales de los países miembros de la CAN.

Es preciso reconocer la importancia económica del comercio electrónico asociado al intercambio de contenidos protegidos por el derecho de autor, como el software, y actualizar la normativa comunitaria andina. En el supuesto de una eventual modificación o sustitución de la norma andina, sería recomendable reemplazar las

28. Por no mencionar las nuevas directrices de la OEP para el examen de invenciones sobre inteligencia artificial. Oficina Europea de Patentes, «Guidelines for Examination», 3.3.1. Artificial intelligence and machine learning, disponible en http://bit.ly/3nOBm5f.

29. Parlamento Europeo, «La propiedad intelectual, industrial y comercial», disponible en http://bit. ly/34DFQ6X. 
referencias a «programas de ordenador» por «programas informáticos»en aras de mayor claridad a efectos de incluir claramente las aplicaciones móviles o apps dentro del ámbito de aplicación de la norma andina.

En ciertas circunstancias, la imposibilidad absoluta de patentar programas informáticos podría parecer inapropiada. Si bien éstos son protegidos automáticamente por el derecho de autor desde su creación, ello no impide la patentabilidad de determinados programas informáticos, bajo condiciones muy estrictas y siempre que se aporte una solución técnica al problema técnico planteado. ${ }^{30}$

Es el caso de las denominadas invenciones implementadas por ordenador, también llamadas «patentes de software», es decir, aquellas invenciones que requieren el uso de un ordenador, red informática u otro aparato programable en los que la ejecución de al menos un programa informático produce un efecto técnico que forma parte de la solución técnica. Es posible encontrar ejemplos de dichas invenciones implementadas por ordenador en edificios inteligentes, vehículos inteligentes (como vehículos autónomos, sistemas de freno, etcétera), sistemas de aire acondicionado, entre otros.

Sin embargo, es importante comentar que, teniendo en consideración los costos asociados a la obtención de una patente, así como la desventaja de divulgar el código fuente, podría ser recomendable recurrir preferentemente a los acuerdos de confidencialidad o al secreto industrial.

Finalmente, cabe destacar que, tanto en el ámbito de la CAN como de la Unión Europea, los videojuegos no se reducen a un mero programa de ordenador, sino que pueden incluir además elementos gráficos y sonoros susceptibles de ser igualmente protegidos por derechos de autor y derechos conexos, dando lugar de esta manera a un conglomerado de obras audiovisuales, musicales, artísticas o literarias que conforman una obra multimedia.

\section{Referencias}

IEEE (1993). Software Engineering Standard: Glossary of Software Engineering Terminology. Nueva York: IEEE Computer Society Press.

INDECOPI (2019). «Aproximación a las invenciones implementadas por ordenador».

Lima: Instituto Nacional de Defensa de la Competencia y de la Protección de la Propiedad Intelectual. Disponible en https://bit.ly/2KyYupp.

TJCA (1999). Interpretación prejudicial recaída en el Proceso 10-IP-99 de 11 de junio de 1999.

-. (2005). Interpretación prejudicial recaída en el Proceso 165-IP-2004 de 6 de abril

30. En Estados Unidos, por el contrario, esta posibilidad parece haberse reducido a partir de la decisión de la Corte Suprema de Estados Unidos de 31 de marzo de 2014 en el caso Alice c/ CLS Bank. 
de 2005, publicada en la Gaceta Oficial del Acuerdo de Cartagena 1195, 11 de mayo de 2005. Disponible en https://bit.ly/2KTxr8I.

-. (2018a). Interpretación prejudicial recaída en el Proceso 464-IP-2017 de 3 de octubre de 2018 (caso Nintendo), publicada en la Gaceta Oficial del Acuerdo de Cartagena 3438, 12 de noviembre de 2018. Disponible en https://bit.ly/2WFd2XD.

-. (2018b). Interpretación prejudicial recaída en el Proceso 242-IP-2017 de 16 de noviembre de 2018, publicada en la Gaceta Oficial del Acuerdo de Cartagena 3472, 5 de diciembre de 2018. Disponible en https://bit.ly/2WGGdcy.

-. (2019). Interpretación prejudicial recaída en el Proceso 569-IP-2018 de 28 de junio de 2019 (caso Hosvital), publicada en la Gaceta Oficial del Acuerdo de Cartagena 3721, 8 de agosto de 2019. Disponible en https://bit.ly/2WKFveG.

\section{Sobre el autor}

Juan Manuel Indacochea Jauregui es master en Derecho de la Propiedad Intelectual por el CEIPI de la Universidad de Estrasburgo, master en Derecho Internacional por la Universidad de Paris-Nanterre, abogado por la Pontificia Universidad Católica del Perú (PUCP), exconsultor del Tribunal de Justicia de la Comunidad Andina (TJCA) y director general de la Sociedad Peruana de Propiedad Intelectual (SPPI). Su correo electrónico es jmindacochea@gmail.com. 
La Revista Tribuna Internacional busca fomentar la reflexión, el debate, el análisis y la comunicación pluralista y con rigor científico en las áreas del derecho internacional público, derecho internacional privado, relaciones internacionales y derecho internacional de los derechos humanos. Los artículos y ensayos son seleccionados mediante revisión de pares externos a la Facultad de Derecho de la Universidad de Chile. Se reciben trabajos en castellano y en inglés.

EDITOR GENERAL

Luis Valentín Ferrada Walker

SITIO WEB

tribunainternacional.uchile.cl

CORREO ELECTRÓNICO

revistatribuna@derecho.uchile.cl

LICENCIA DE ESTE ARTÍ́CULO

Creative Commons Atribución Compartir Igual 4.o Internacional

La edición de textos, el diseño editorial

y la conversión a formatos electrónicos de este artículo

estuvieron a cargo de Tipográfica

(www.tipografica.io) 